\title{
Biodiversité structurelle et fonctionnelle des peuplements de macro-invertébrés en tant que descripteur de la variabilité hydraulique : exemple de deux parties court-circuitées du Rhône moyen \\ Biodiversity of macroinvertebrate communities as describer of hydraulic variabiliy: the example of two by-passed sections of the Middle Rhône River
}

\author{
J. F. Fruget et J. Dessaix
}

Volume 15, numéro 1, 2002

URI : https://id.erudit.org/iderudit/705447ar

DOI : https://doi.org/10.7202/705447ar

Aller au sommaire du numéro

Éditeur(s)

Université du Québec - INRS-Eau, Terre et Environnement (INRS-ETE)

ISSN

0992-7158 (imprimé)

1718-8598 (numérique)

\section{Découvrir la revue}

Citer cet article

Fruget, J. F. \& Dessaix, J. (2002). Biodiversité structurelle et fonctionnelle des peuplements de macro-invertébrés en tant que descripteur de la variabilité hydraulique : exemple de deux parties court-circuitées du Rhône moyen. Revue des sciences de l'eau / Journal of Water Science, 15(1), 209-221.

https://doi.org/10.7202/705447ar

\section{Résumé de l'article}

Le peuplement faunistique d'un hydrosystème est constamment soumis à la variabilité spatio-temporelle de l'environnement. Il en résulte que les espèces dominantes sont celles présentant les caractéristiques biologiques et les préférendums écologiques les plus appropriés aux différents types d'habitats de la mosaïque fluviale. Une approche multiparamétrique de description de l'état physique du milieu à partir de la prise en compte simultanée d'indicateurs de structure et de fonction des communautés d'invertébrés a été testée sur le Rhône Moyen. L'étude de quatre stations physiquement dissemblables situées dans deux secteurs court-circuités montre ainsi qu'à la structuration selon la vitesse du courant (unités fonctionnelles) correspond des différences trophiques et écologiques, tandis qu'à la structuration physique du milieu (secteur de cours d'eau) correspond des différences taxonomiques. La disponibilité en habitats pour les invertébrés est alors corrélée à la configuration du chenal du tronçon considéré. Les assemblages d'espèces répondent ainsi, au travers de leur occurrence et de leur abondance, à un gradient physique. Cette approche, prédictive et appliquée, mettant en parallèle constat biologique et structure physique des stations et des milieux, permet d'établir une typologie fonctionnelle des écosystèmes aquatiques considérés et de proposer une gestion intégrée adaptée aux problèmes posés (gestion des habitats et de leur biodiversité, restauration hydraulique et écologique des milieux, etc.). 


\title{
Biodiversité structurelle et fonctionnelle des peuplements de macroinvertébrés en tant que descripteur de la variabilité hydraulique : exemple de deux parties court-circuitées du Rhône Moyen
}

\author{
Biodiversity of macroinvertebrate communities \\ as describer of hydraulic variabiliy: the example \\ of two by-passed sections of the Middle Rhône River
}

\author{
J.-F. FRUGET ${ }^{1,2}{ }^{*}$, J. DESSAIX ${ }^{1,2}$
}

The aquatic communities of a hydrosystem are constantly subject to the space-time variability of the environment. As a result, the dominant species are those presenting the biological characteristics and the ecological preferences best adapted with the various types of habitats of the river mosaic. $A$ multiparametric description of physical structure from macroinvertebrate communities, taking into account both indicators of structure and functioning of these communities, was tested on the Middle Rhône River (France). The study of four physically dissimilar stations located in two by-passed sections showed that the arrangement due to the current velocity (functional units) corresponded to trophic and ecological differences, whereas the arrangement due to physical conditions (reach of river) corresponded to taxonomic differences. The availability in habitats for the invertebrates was correlated with the configuration of the channel of the section considered. The patches of species answered thus, through their occurrence and their abundance, to a physical gradient. This approach, predictive and applied, with parallel between the biological report and the physical structure of the stations and the environments, makes it possible to establish a functional typology of the aquatic ecosystems considered and to propose an integrated management adapted to the problems arising (management of the habitats and of their biodiversity, hydraulic and ecological restoration of the environments, etc).

Key-words: invertebrates, biodiversity, habitat, Rhône river.

1. ARALEP, Écologie des eaux douces, Domaine scientifique de la Doua, Bât. CEl, BP 2132, 69603 Villeurbanne, France.

2. UMR CNRS 5023, Écologie des hydrosystèmes fluviaux, Université Lyon 1, 69622 Villeurbanne, France.

* Correspondance. E-mail : jean-francois.fruget@univ-lyont.fr

Les commentaires seront reçus jusqu'au 31 mars 2003. 


\section{RÉSUMÉ}

Le peuplement faunistique d'un hydrosystème est constamment soumis à la variabilité spatio-temporelle de l'environnement. Il en résulte que les espèces dominantes sont celles présentant les caractéristiques biologiques et les préférendums écologiques les plus appropriés aux différents types d'habitats de la mosaïque fluviale. Une approche multiparamétrique de description de l'état physique du milieu à partir de la prise en compte simultanée d'indicateurs de structure et de fonction des communautés d'invertébrés a été testée sur le Rhône Moyen. L'étude de quatre stations physiquement dissemblables situées dans deux secteurs court-circuités montre ainsi qu'à la structuration selon la vitesse du courant (unités fonctionnelles) correspond des différences trophiques et écologiques, tandis qu'à la structuration physique du milieu (secteur de cours d'eau) correspond des différences taxonomiques. La disponibilité en habitats pour les invertébrés est alors corrélée à la configuration du chenal du tronçon considéré. Les assemblages d'espèces répondent ainsi, au travers de leur occurrence et de leur abondance, à un gradient physique. Cette approche, prédictive et appliquée, mettant en parallèle constat biologique et structure physique des stations et des milieux, permet d'établir une typologie fonctionnelle des écosystèmes aquatiques considérés et de proposer une gestion intégrée adaptée aux problèmes posés (gestion des habitats et de leur biodiversité, restauration hydraulique et écologique des milieux, etc.).

Mots clés : invertébrés, biodiversité, habitat, Rhône.

\section{1 - INTRODUCTION}

Le peuplement faunistique d'un hydrosystème est constamment soumis à la variabilité spatio-temporelle de l'environnement. II en résulte que les espèces dominantes sont celles présentant les caractéristiques biologiques et les préférendums écologiques les plus appropriés aux différents types d'habitats de la mosaïque fluviale (TOWNSEND, 1989 ; TOWNSEND et HILDREW, 1994). Les variations observées dans la structure des peuplements dépendent donc des modifications des caractéristiques environnementales. L'étude conjointe de la composition taxonomique et de la signification biologique et écologique des peuplements d'une part, la description des conditions physiques du milieu d'autre part, permettent la compréhension de certains mécanismes expliquant la présence ou l'absence de différents groupes d'espèces et ainsi la prédiction de l'évolution des peuplements aquatiques.

Ce dernier point est particulièrement intéressant dans le cadre du programme décennal de restauration hydraulique et écologique du Rhône, mis en place récemment, qui vise à réhabiliter le fleuve et ses milieux annexes, en particulier par la révision des débits réservés et la restauration des connectivités longitudinales et transversales.

Cet article vise donc à valider une approche multiparamétrique de description de l'état physique du milieu à partir de la prise en compte simultanée d'indicateurs de structure et de fonction des communautés d'invertébrés. 


\section{2 - SITE D’ÉTUDE - MATÉRIEL ET MÉTHODES}

Les aménagements hydroélectriques de Pierre-Bénite et de Péage-deRoussillon (figure 1), en aval de Lyon, sont suivis depuis plus de dix ans dans le cadre de diverses études (FRUGET, 1991 ; FRUGET et al., 1999 ; ARALEPBP, 2000). Quatre stations physiquement dissemblables, deux d'entre elles étant situées en secteurs de radiers et deux autres en secteurs lentiques endigués (figure 1A), régulièrement échantillonnées, sont considérées dans le cadre de cette étude. Actuellement, et en dehors des périodes de hautes eaux, ces milieux reçoivent un débit réservé qui varie entre 10 et $20 \mathrm{~m}^{3} / \mathrm{s}$ (automne-hiver - printemps-été), pour un débit moyen total du Rhône à ce niveau d'environ $1030 \mathrm{~m}^{3} / \mathrm{s}$. Lors des forts épisodes hydrologiques, l'excédent de débit non turbiné par les usines hydroélectriques est déversé via le barrage dans la partie court-circuitée qui joue alors le rôle d'évacuateur de crues, le débit pouvant ainsi être multiplié par 10 ou 100 en quelques heures (figure 1B).

Les prélèvements faunistiques sont réalisés d'une part par dragages dans le chenal, et d'autre part selon la technique dite des microhabitats (BOURNAUD et COGERINO, 1986 ; COGERINO, 1989) au niveau des rives. Des relevés de différentes variables mésologiques relatives à l'habitat (profondeur, vitesse du courant, type de substrat échantillonné) sont réalisés parallèlement. Les dragages sont effectués à l'aide d'une drague triangulaire de type irlandais, remorquée à partir d'une embarcation à moteur, lorsque la profondeur est supérieure à $1 \mathrm{~m}$ (stations endiguées). En-deçà de cette profondeur (stations radiers), les prélèvements sont effectués avec un filet de type Surber. Le vide de maille de la drague et du Surber est de $500 \mu$. Au niveau des rives, la macrofaune est récoltée par prélèvement direct du microhabitat à l'aide d'un filet de type Surber. Les divers types de microhabitats (substrats durs, herbiers, sédiments fins), en réalité les mésohabitats sensu ARMITAGE et al. (1995), sont prospectés en fonction de leur occurrence. La zone rivulaire prospectée correspond à la rive immergée pour un débit faible à moyen, pour une distance horizontale de $1 \mathrm{~m}$ à partir de la berge.

Les prélèvements faunistiques (et les relevés des variables d'habitat correspondants) sont effectués le long d'un transect transversal par rapport à l'axe du chenal : au niveau des microhabitats dominants de chacune des rives et au centre du chenal. L'emploi combiné de plusieurs techniques d'échantillonnage, associé à la description physique du milieu, permet d'appréhender l'hétérogénéité physique et faunistique, tant transversale (rive-chenal) que longitudinale (chenal lentique endigué-radiers lotiques). Le substrat ainsi récolté et les invertébrés associés sont stockés et conservés dans une boîte contenant du formol à $4 \%$. Ils sont ensuite triés au laboratoire, conservés dans de l'alcool à $70 \%$ et déterminés à l'espèce le plus souvent possible, hormis pour les Oligochètes et les Diptères en raison de difficultés systématiques (famille ou sous-famille pour ces deux groupes faunistiques).

USSEGLIO-POLATERA et al. (2000) ont développé un nouvel outil de mesure de la diversité fonctionnelle des peuplements benthiques au travers d'une approche originale basée sur la prise en compte des mécanismes (fonctionnement) conduisant à la présence ou à l'absence de certaines espèces dans le milieu. Nous avons donc utilisé cette approche fonctionnelle en complément 


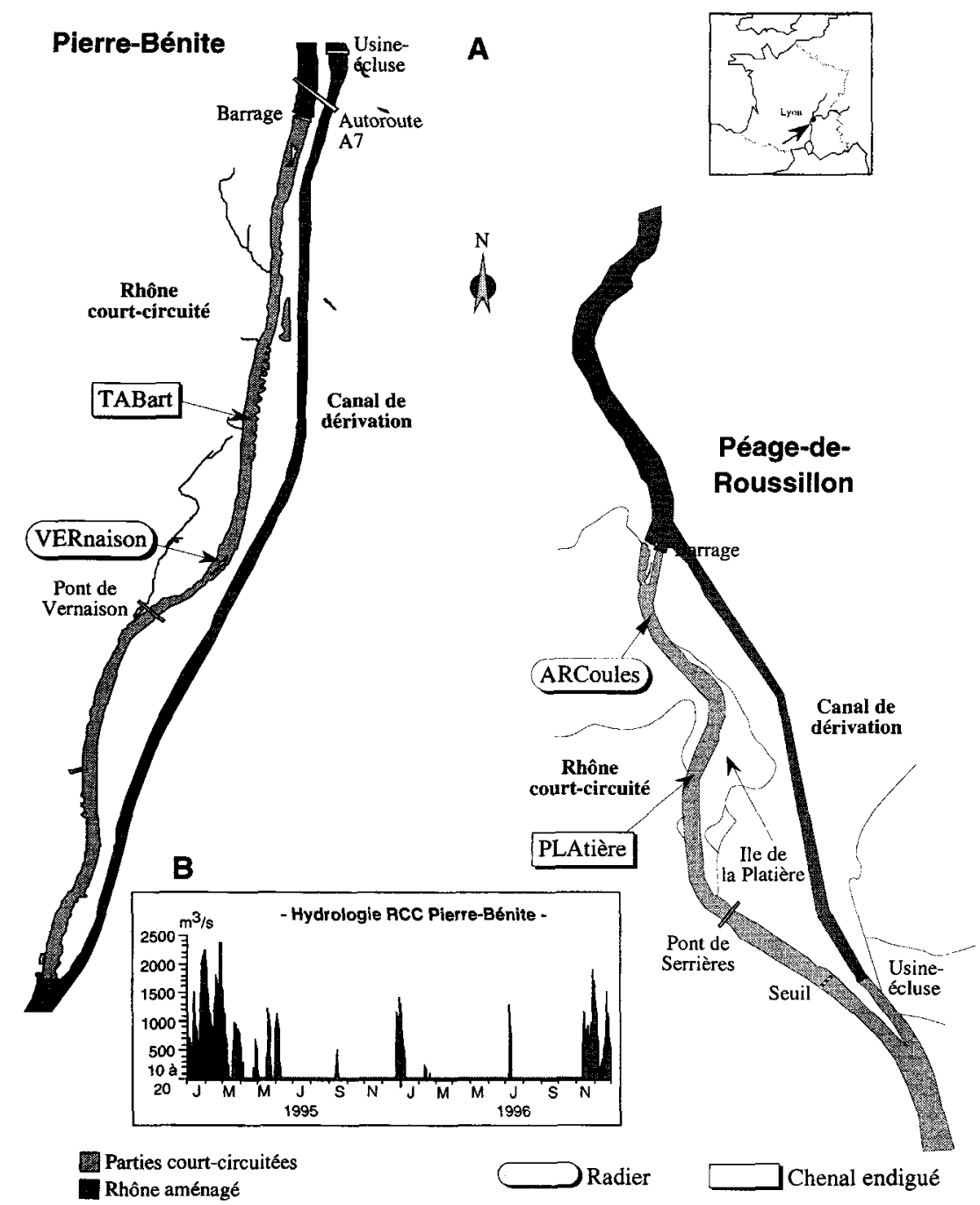

Figure 1A Localisation des stations d'étude au niveau des deux parties courtcircuitées du Rhône Moyen considérées.

B Exemple du fonctionnement hydrologique du Rhône court-circuité de Pierre-Bénite en 1995-96.

A Location of the stations studied in the two by-passed sections considered on the Middle Rhône.

$B$ Example of the hydrological functioning of Pierre-Benite by-passed section in 1995-96.

du calcul des paramètres de communautés classiques (richesse, diversité, etc.) et des analyses multivariées des matrices de données visant à caractériser la structure des communautés d'invertébrés. Ce nouvel outil est basé sur la définition de groupes d'espèces possédant des exigences biologiques et écolo- 
giques similaires, d'une part au travers de la distinction de huit groupes biologiques (en particulier discriminés par les critères de taille, de stratégie reproductive, de source de nourriture et de mode d'alimentation), d'autre part au travers de l'identification de sept groupes écologiques (principalement définis par le type d'habitat).

\section{3 - RÉSULTATS}

\subsection{Variables mésologiques et hydrauliques}

Outre la description de l'habitat propre aux invertébrés échantillonnés mentionnée précédemment, l'environnement hydraulique des stations a pu être décrit par les données mésologiques (figure $2 A$ ) recueillies à l'occasion d'une étude visant à simuler les capacités d'accueil potentielles pour la faune aquatique, afin de prévoir l'évolution de l'habitat entre la situation actuelle avec un débit réservé de 10 à $20 \mathrm{~m}^{3} / \mathrm{s}$ et une situation ultérieure à $100 \mathrm{~m}^{3} / \mathrm{s}$, par exemple (CEMAGREF, 1997). La turbulence, exprimée par le nombre de Froude, différencie les deux types d'unités fonctionnelles (chenal lentique endigué vs radier lotique), tandis que l'énergie cinétique (décrivant l'écoulement), exprimée par le nombre de Reynolds, différencie les deux aménagements (Pierre-Bénite vs Péage-de-Roussillon), en particulier sur la base de la structure du chenal (figure 2B).

\subsection{Structure faunistique}

Un total de 85 taxons a été récolté dans le cadre du protocole d'échantillonnage retenu, respectivement 51 et 49 sur les radiers de Vernaison et d'Arcoules, 61 et 41 dans les secteurs endigués de l'île Tabard et de l'île de la Platière. L'analyse de la structure spatiale des peuplements benthiques a été réalisée par AFC interclasses à partir d'un jeu de données regroupant 79 taxons et 75 relevés unitaires, du double point de vue stations et habitats (figure 3). Pour cela, les taxons "rares » sur le critère d'abondance, c'est-à-dire $\leq 2$ individus au total ont été éliminés, mais certains taxons "juvéniles " ou " nymphes " ont été distingués.

L'axe 1 de l'analyse inter-stations (figure $3 A$ ) distingue les deux aménagements : présence de taxons potamiques rhéophiles à Pierre-Bénite tels que Theodoxus fluviatilis, Heptagenia sulphurea, Hydropsyche spp., Psychomyia pusilla ; taxons globalement plus potamo-lénitiques à Péage-de-Roussillon parmi lesquels Asellus aquaticus, Dreissena polymorpha, Ceraclea dissimilis, Tinodes waeneri. L'axe 2 différencie les radiers par rapport aux mouilles endiguées, en particulier avec la présence de taxons épipotamiques dans les premiers tels qu'Ancylus fluviatilis, Baetis fuscatus, Heptagenia sulphurea, Hydropsyche spp.

Le plan factoriel $1 \times 2$ de l'analyse inter-habitats (figure $3 B$ ) est globalement défini par quelques habitats peu représentés ( 3 à 4 relevés pour chacun d'eux) et présentant certains taxons plus spécifiques : l'axe 1 oppose les graviers 
A - Variables mésologiques
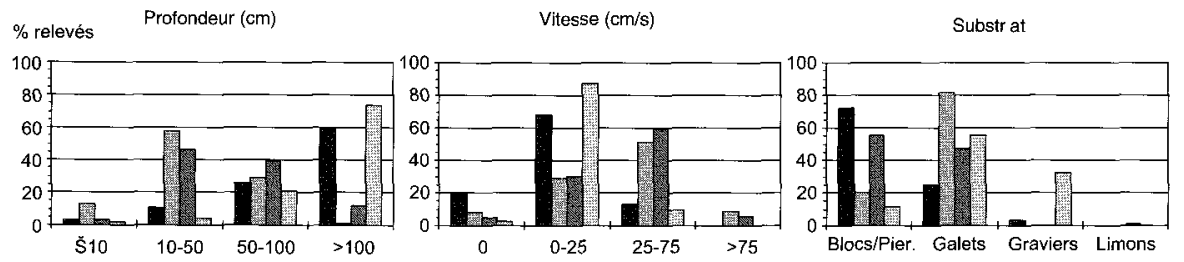

TABart 술 VERnaison 圈 ARCoules :

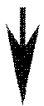

B - Variables hydrauliques

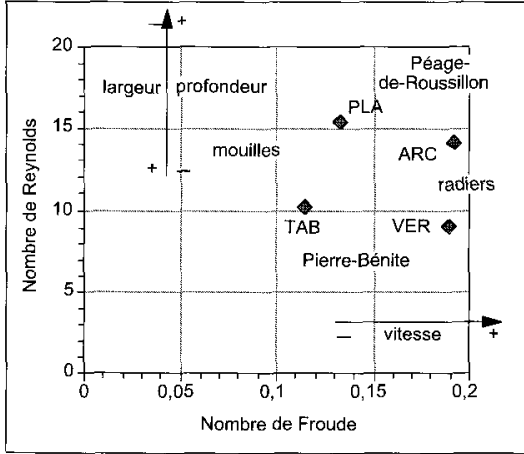

Figure 2 Répartition des modalités des différentes variables mésologiques mesurées lors de la description des conditions physiques de chaque station $(A)$, et représentation des variables hydrauliques propres à chacune d'elle calculées pour le débit Q20 (B).

Distribution of habitat variables measured during the description of physical conditions of each station $(A)$, and representation of hydraulic variables of each one calculated for the Q20 discharge $(B)$.

(présents à l'île Tabard) au couple macrophytes/algues tandis que l'axe 2 oppose les graviers au couple dalles/branches, voire limons/sables (présents à l'île de la Platière). Ces habitats sont tous essentiellement récoltés dans la gamme de vitesse nulle, hormis le couple macrophytes/algues qui présentent quelques relevés (renoncules, fontinales) dans la gamme de vitesse moyenne (25 à $75 \mathrm{~cm} / \mathrm{s}$ ). Les taxons les plus représentatifs sont Caenis luctuosa et Corbicula fluminea pour les graviers, Baetis fuscatus pour les macrophytes et les algues, ainsi que Cloeon dipterum, Ephemerella ignita, Hydroporus sp. et Laccobius sp. pour ces dernières, Tinodes waeneri pour les dalles et les branches. Quant aux limons et aux sables, ce sont deux habitats extrêmement pauvres tant en termes de richesse spécifique que d'abondance, essentiellement dominés par les Oligochètes autres que les Naididés et quelques Diptères Chironomidés. 

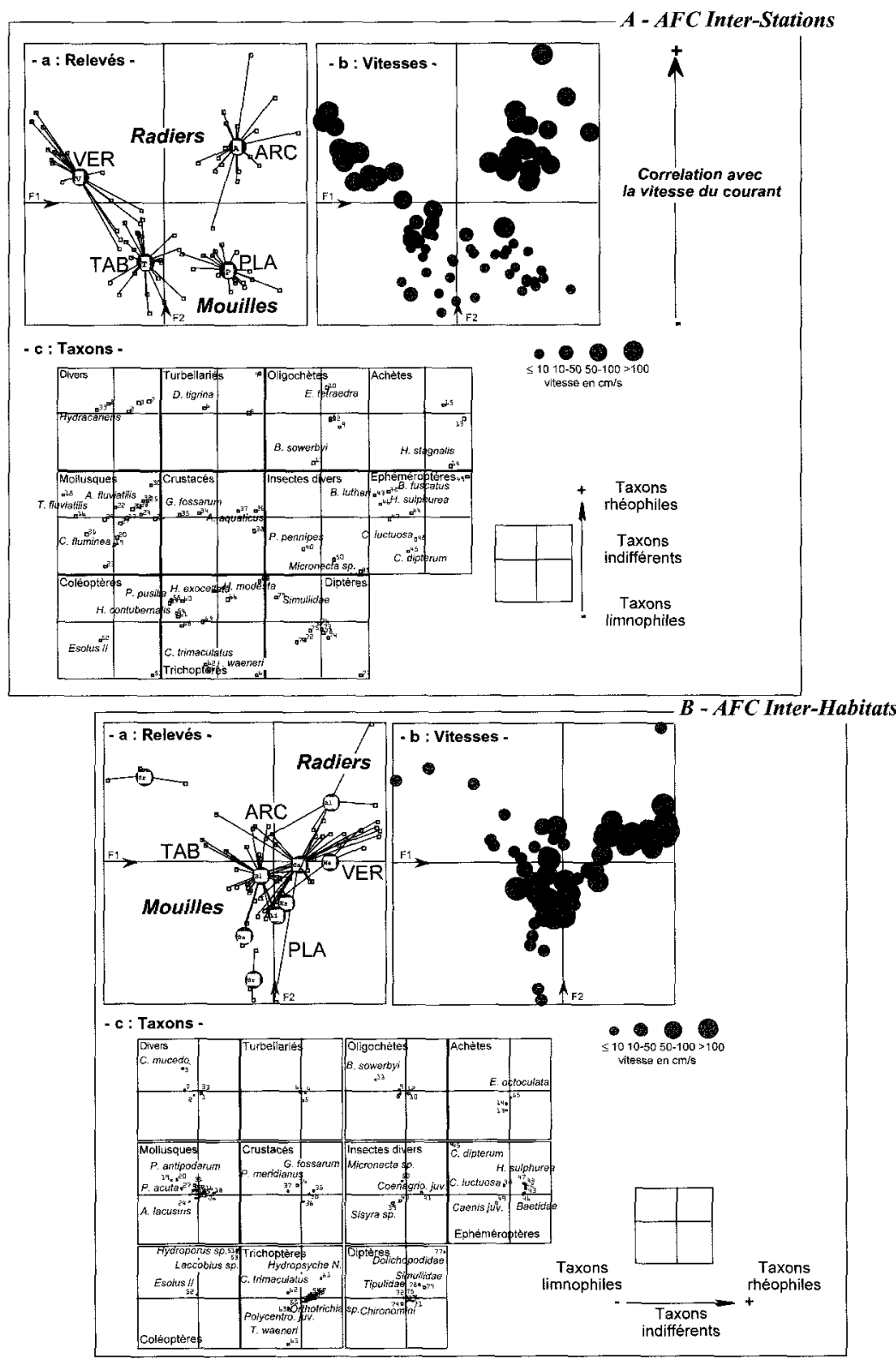

Figure 3 Analyses inter-classes des peuplements benthiques selon le double point de vue stations (A) et habitats (B).

Pour chaque analyse, la carte factorielle des relevés, la projection de la vitesse du courant de chaque relevé et la carte factorielle des taxons sont représentées sur le plan F1 $\times$ F2.

Between-class analyses of the benthic communities from the two point of view stations $(A)$ and habitats $(B)$.

For each analysis, the factorial map of samples, the superposition of the current velocity of each sample and the factorial map of taxa are shown. 


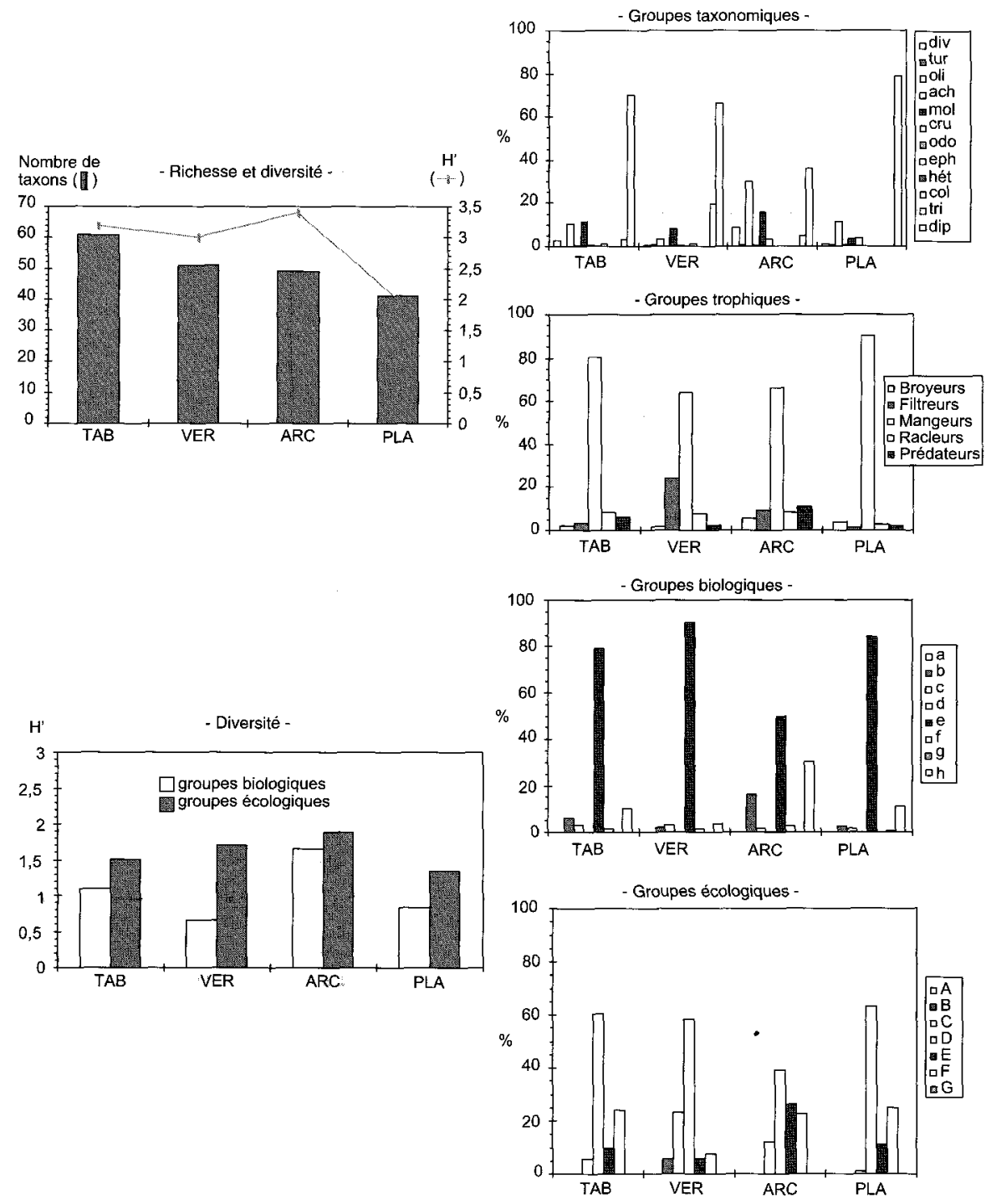

Figure 4 Caractéristiques écologiques des communautés d'invertébrés des quatre stations échantillonnées.

Ecological characteristics of invertebrate communities of the four sampled stations.

\subsection{Caractéristiques bioécologiques des communautés d'invertébrés}

Si les groupes taxonomiques dominants (abondance $>5 \%$; Diptères, Oligochètes et Mollusques), voire subdominants (abondance comprise entre 2 et $5 \%$; Trichoptères, Crustacés et taxons « divers ") sont les mêmes entre les quatre stations (figure 4), c'est-à-dire entre aménagements d'une part et unités 
fonctionnelles d'autre part, quelques particularités peuvent toutefois être signalées: les Mollusques Bivalves sont essentiellement représentés par Corbicula fluminea à Pierre-Bénite tandis que ce sont les Sphaeridés et Dreissena polymorpha qui dominent à Péage-de-Roussillon ; les Trichoptères sont globalement plus nombreux à Pierre-Bénite (Cyrnus et Ecnomus dans la partie endiguée, Hydropsyche sur le radier) ; les Crustacés sont significativement présents à Péage-de-Roussillon grâce aux Aselles ; les taxons « divers " sont bien représentés à Arcoules par les Hydres.

Découlant de la forte abondance des Diptères et des Oligochètes, les mangeurs de sédiment constituent le groupe trophique dominant (figure 4). Les filtreurs sur les radiers (Trichoptères Hydropsychidés, Mollusques Bivalves et Diptères Simuliidés), les broyeurs à Arcoules (Asellus aquaticus et Ceraclea dissimilis), les racleurs et brouteurs de substrat aux différentes stations (Gastéropodes) et les prédateurs à l'île Tabard et Arcoules (Hydres) atténuent plus ou moins cette forte dominance. Le régime alimentaire des peuplements invertébrés des différentes stations est ainsi à forte dominante détritivore, voire omnivore, en accord avec une charge organique et une productivité importantes à l'origine de la présence de débris de toutes sortes (débris et sédiments fins, microphytes, etc.).

Les groupes biologiques, tels que définis par USSEGLIO-POLATERA et al. (2000), montrent que l'essentiel des individus appartiennent aux groupes e et $h$ (figure 4). Le groupe e, nettement dominant, abrite des espèces de taille petite à moyenne, à courte durée de vie, uni- ou plurivoltines, souvent nageuses, détritivores, à mode d'alimentation broyeur-fragmenteur ou racleur-brouteur, telles que les Gastéropodes, les Aselles, les Hydropsyche, les Baetidés ou les Chironomidés. Le groupe $\mathrm{h}$ correspond essentiellement aux Oligochètes, avec des espèces plurivoltines, mangeuses de sédiment fin, détritivores. À l'île Tabard, et plus encore à Arcoules, le groupe $b$ apparaît également de manière significative, avec des taxons de taille moyenne à grande, nageurs ou fouisseurs, à nourriture et habitat variés, tels que les Hydres, les Sangsues, les Bivalves et les Gammares.

Les groupes écologiques, toujours au sens d'USSEGLIO-POLATERA et al. (2000), apparaissent plus diversifiés avec de 3 à 4 groupes dominants, souvent variables entre les stations (figure 4). Si la différence inter-aménagements est peu marquée, avec 4 groupes globalement présents en mêmes proportions à Pierre-Bénite et à Péage-de-Roussillon (D-F puis E-C), il en va différemment entre les deux unités fonctionnelles où le nombre de groupes écologiques significativement représentés est moindre dans les stations des mouilles endiguées $(D-F$, voire $E)$ par rapport à celles des radiers courants $(D-F$, voire $E$, plus $\mathrm{C}$, voire $\mathrm{B})$. Ces groupes sont définis par les principaux taxons et caractéristiques suivantes:

- Groupe D : taxons vivant près des rives, en milieu plutôt lentique, eurythermes, oligo- à $\beta$-mésosaprobes, à large préférendum quant au substrat, tels que les Chironomes et divers taxons des genres Pisidium, Chaetogaster, Glossiphonia et Erpobdella, par exemple. Ce groupe abrite de 40 à $60 \%$ des individus selon la station considérée ;

- Groupe F : taxons d'habitat lentique, en particulier les macrophytes, parmi lesquels on rencontre Stylaria lacustris, Helobdella stagnalis, Asellus aquaticus, Cloeon dipterum, Caenis luctuosa, Cyrnus trimaculatus, en par- 
ticulier. Ce groupe représente environ $1 / 4$ des effectifs, hormis à Vernaison ;

- Groupe E : taxons eurythermes, $\beta$ à $\alpha$-mésosaprobes, vivants dans différents types d'habitats rivulaires lentiques, tels que divers Oligochètes Naididés et Tubificidés, Potamopyrgus antipodarum, Corbicula fluminea, Dreissena polymorpha, Platycnemis pennipes, Ceraclea dissimilis, Ecnomus tenellus. Les effectifs de ce groupe sont variables selon la station : ils sont similaires entre les deux stations mouilles mais très supérieurs à Péage-de-Roussillon en ce qui concerne les stations radiers ;

- Groupe $\mathbf{C}$ : taxons rhithroniques à épipotamiques, eurythermes, oligo- à $\beta$-mésosaprobes, vivants sur un substrat minéral ou sur la végétation, avec des vitesses de courant faibles à moyennes, tels que Hydra s.l., Ancylus fluviatilis, Theodoxus fluviatilis, Gammarus fossarum, Hydropsyche spp., Psychomyia pusilla, Baetis fuscatus, Heptagenia sulphurea. Ce groupe est particulièrement abondant aux deux stations radiers ;

- Groupe B : taxons rhithroniques, oligosaprobes, eurythermes, rhéophiles, vivants sur un substrat minéral grossier ou sur la végétation, essentiellement représentés par Esolus parallelepipedus et surtout les Simulies. II est abondamment présent sur le radier de Vernaison par l'intermédiaire de ce dernier taxon.

\section{4 - DISCUSSION}

En mettant en parallèle les résultats faunistiques et les résultats mésologiques et hydrauliques, trois constats peuvent être dressés :

(i) L'axe de structuration selon la vitesse du courant, différenciant les deux unités fonctionnelles (figure 2), correspond à un axe de différences trophiques et écologiques où, à un pool de taxons à exigences communes, s'ajoutent sur les radiers des espèces plus rhéophiles, rhithroniques à épipotamiques, nécessitant un substrat minéral plus grossier, à mode d'alimentation filtreur.

(ii) À l'inverse, l'axe de structuration selon la structure physique, différenciant les deux aménagements (figure 2), apparaît être un axe de différences taxonomiques avec la présence de quelques espèces potamiques rhéophiles à Pierre-Bénite (influence possible d'apports faunistiques en provenance du Haut-Rhône, partie court-circuitée protégée des rejets et apports se produisant dans le canal de dérivation), tandis que le peuplement est globalement plus potamo-lénitique à Péage-de-Roussillon (en aval des différents secteurs de rejets domestiques et industriels de l'agglomération lyonnaise et du couloir de la chimie, charge organique et eutrophisation plus marquées).

(iii) Concernant l'arrangement de la mosaïque fluviale, si une similarité d'habitat principal existe entre les deux parties court-circuitées quelle que soit l'unité fonctionnelle et la station (mésohabitats minéraux grossiers de types blocs et galets associés à une végétation macrophytique et algale - renoncules et fontinales sur les radiers, potamot pectiné et algues filamenteuses dans les 
mouilles endiguées), les caractéristiques de l'écoulement influent par la suite sur l'habitat secondaire, en particulier sur les mésohabitats minéraux fins que sont par exemple les graviers à l'île Tabard ou les limons et sables à l'île de la Platière (figure 3). Ces derniers habitats sont très pauvres tant qualitativement que quantitativement. Les modifications du fonctionnement et des conditions hydrauliques auront donc prioritairement un effet sur ces constituants secondaires de la mosaïque fluviale.

A l'échelle du Rhône en aval de Lyon, les taxons indicateurs ainsi que les taxons dominants sont globalement caractéristiques d'un milieu potamo-lénitique (FRUGET, 1991). Dans ce cadre, l'influence de l'hydrodynamique dans la répartition des espèces est la variable clé du fonctionnement de l'hydrosystème, la disponibilité en habitats pour les invertébrés étant corrélée à la configuration du chenal du tronçon considéré. La présence de différents taxons potamiques réellement rhéophiles au niveau des différents radiers le confirme. L'existence de ces taxons atteste donc de potentialités toujours effectives du milieu, ne demandant qu'à s'exprimer mais, inversement, pouvant également être rapidement inhibées. L'amélioration des conditions hydrodynamiques doit donc tendre vers une restauration de l'état initial, tant du point de vue physique (cours d'eau de plaine à substrat grossier, turbulence importante et température relativement fraîche) que biologique (espèces épipotamiques). Or, certains taxons potamiques et/ou rhéophiles sont encore présents mais en faibles effectifs et de façon localisée. Plus que l'apparition de taxons spécifiques, on peut donc penser que l'augmentation de débit projetée dans le cadre du plan décennal Rhône, en premier lieu à PierreBénite, permettra une plus large répartition de ces taxons, ainsi qu'une augmentation de leurs effectifs. L'amélioration des conditions hydrodynamiques pourrait également permettre à des taxons sensibles arrivant par dérive lors des crues de s'installer durablement et d'augmenter ainsi la diversité biologique de ces milieux.

II ressort de cette étude que les assemblages d'espèces répondent, au travers de leur occurrence et de leur abondance, à un gradient physique. Ces assemblages peuvent être considérés comme des structures en mosaïque, dynamiques dans l'espace et dans le temps. Le constat biologique a pu ainsi être mis en parallèle avec la structure physique des stations et des milieux considérés. Toutefois, si des courbes de préférences d'habitat ont été développées pour différentes espèces (GORE et JUDY, 1981 ; ORTH et MAUGHAN, 1983 ; JOWEIT et RICHARDSON, 1990 ; etc.), il existe peu de modèles quantitatifs reliant les peuplements d'invertébrés à l'habitat physique. Au travers d'une étude sur l'influence discriminante des facteurs vitesse, hauteur d'eau et substrat sur les densités et biomasses globales du benthos dans des cours d'eau salmonicoles, WASSON et al. (1995) ont tenté une ébauche de modèles prédictifs des relations habitat/invertébrés, dans la perspective de les intégrer dans des modèles habitat/poissons. L'utilisation d'une approche multiparamétrique rentre en partie dans cette problématique. Nos résultats obtenus sur le Rhône-Moyen confirment par exemple les travaux d'ARMITAGE (ARMITAGE et al., 1995 ; ARMITAGE et PARDO, 1995) :

(i) L'étude conjointe de la distribution des mésohabitats (variable selon le contexte physique et hydraulique) et des communautés d'invertébrés benthiques associées permet de caractériser les résultats obtenus en termes d'impact lors d'opérations d'aménagement (modifications de débit, dragages, etc.).

(ii) L'échelle du mésohabitat se révèle pertinente pour l'étude des peuplements d'invertébrés en terme de gestion, en particulier le substrat minéral per- 
met l'intégration de l'histoire géologique, du régime hydrologique et des divers caractéristiques physiques du chenal étudié.

(iii) La connaissance de la faune associée permet alors d'évaluer le degré de résilience du cours d'eau après perturbation et les possibilités futures de restauration. Par la suite, les liens possibles entre les compartiments physiques et biologiques peuvent être testés et discutés à plus large échelle (passage du tronçon au cours d'eau, par ex.).

En conclusion, une telle approche, prédictive et appliquée, permet d'établir une typologie fonctionnelle des écosystèmes aquatiques considérés et de proposer une gestion intégrée adaptée aux problèmes posés (gestion des habitats et de leur biodiversité, restauration hydraulique et écologique des milieux, etc.). Dans l'absolu, la combinaison de modèles de simulation du couple vitessehauteur d'eau et de modèles de transport solide devrait permettre la prédiction des types d'habitats rencontrés et des changements dans leur distribution en fonction du type et du niveau de perturbation, et donc de l'évolution, tant qualitative que quantitative, de la faune associée. C'est l'objectif que nous nous sommes assignés dans le cadre de notre participation au programme décennal «Rhône », avec un suivi scientifique s'articulant autour des trois aspects (i) évolution des peuplements, (ii) validation des prédictions, (iii) identification de métriques biologiques et physiques pertinentes, en particulier au travers de la simulation de modèles d'habitats (LAMOUROuX Coord., 2000).

\section{RÉFÉRENCES BIBLIOGRAPHIQUES}

ARALEPBP, 2000. Suivi de l'incidence de l'augmentation du débit réservé dans le Vieux-Rhône de Pierre-Bénite. Étude de la faune benthique avant augmentation. Synthèse 1995-1999. Rapport à la Compagnie Nationale du Rhône, Lyon, $10 \mathrm{p}$.

ARMITAGE P.D., PARDO I. 1995. Impact assessment of regulation at the reach level using macroinvertebrate information from mesohabitats. Regul. Rivers, 10, 147-158.

ARMITAGE P.D., PARDO I., BROWN A., 1995. Temporal constancy of faunal assemblages in "mesohabitats". Application to management? Arch. Hydrobiol., 133 (3), 367-387.

BOURNAUD M., COGERINO L., 1986. Les microhabitats aquatiques des rives d'un grand cours d'eau : approche faunistique. Annls Limnol., 22 (3), 285-294.

CEMAGREF, 1997. Chute de Pierre-Bénite. Suivi de l'incidence de l'augmentation du débit réservé dans le Vieux-Rhône. Rapport à la Compagnie Nationale du Rhône, Lyon, $139 \mathrm{p}$.
COGERINO L., 1989. Les rives aquatiques des grands cours d'eau : caractérisation mésologique et faunistique. Th. Doct. Univ. Lyon l, $329 \mathrm{p}$.

FRUGET J.F., 1991. The impact of river regulation on the lotic macroinvertebrate communities of the Lower Rhône, France. Regul. Rivers, 6 (4), 241-255.

FRUGET J.F., CENTOFANTI M., DESSAIX J., OLIVIER J.M., DRUART J.C., MARTINEZ P.J., 1999. Synthèse des dix premières années de suivi hydrobiologique du Rhône au niveau de la centrale nucléaire de Saint-Alban. Hydroécol. Appl., 11 (1-2), 29-69.

GORE J.A., JUDY R.D. Jr. 1981. Predictive models of benthic macroinvertebrate density for use in instream flow and regulated flow management. Can. J. Fish. Aquat. Sci., 38, 1363-1370.

JOWETT I.G., RICHARDSON J., 1990. Microhabitat preferences of benthic invertebrates in a New Zealand river and the development of instream flow-habitat models for Deleatidium spp. New Zea- 
land J. Marine Freshwater Research, 24, 19-30.

LAMOUROUX N. (Coord.) 2000. Suivi scientifique du Vieux-Rhône de Pierre-Bénite. Proposition technique. Document CEMAGREF Lyon, Division BEA.

ORTH D.J., MAUGHAN O.E. 1983. Microhabitat preferences of benthic fauna in a woodland stream. Hydrobiologia, 106 (2), 157-168.

TOWNSEND C.R. 1989. The patch dynamic concept of stream community ecology. J. North Am. Benthol. Soc., 8, 36-50.

TOWNSEND C.R., HILDREW A.G., 1994. Species traits in relation to a habitat tem- plet for river systems. Freshwat. Biol., 31, 265-275.

USSEGLIO-POLATERA P., BOURNAUD M., RICHOUX P., TACHET H., 2000. Biologi$\mathrm{Cal}$ and ecological traits of benthic freshwater macroinvertebrates: relationships and definition of groups of similar traits. Freshwat. Biol., 43, 175-205.

WASSON J.G., BONNARD R., MARIDET L., 1995. Réponses globales des invertébrés benthiques aux conditions d'habitat physique dans des cours d'eau salmonicoles : perspectives d'intégration dans des modèles habitat/poissons. Bull. Fr. Pêche Piscic., 337-338$339,291-299$. 